\title{
Effect of the Pre-Treatment Severity on the Antioxidant Properties of Ethanol Organosolv Miscanthus $x$ giganteus Lignin
}

\author{
Roland El Hage ${ }^{1,2}$, Dominique Perrin ${ }^{3}$, Nicolas Brosse ${ }^{3 *}$ \\ ${ }^{1}$ Centre des Matériaux de Grande Diffusion (CMGD), Ecole des Mines d'Alès, France; ${ }^{2}$ INNOBAT, Cap Alpha, Clapiers, France; \\ ${ }^{3}$ Laboratoire d'Etude et de Recherche sur le MAteriau Bois, Faculté des Sciences et Techniques, Nancy-Université, Vandœuvre- \\ lès-Nancy, France. \\ Email: *Nicolas.Brosse@1ermab.uhp-nancy.fr
}

Received November $7^{\text {th }}, 2011$; revised December $29^{\text {th }}, 2011$; accepted January $8^{\text {th }}, 2012$

\begin{abstract}
The effect of the severity of an organosolv treatment of Miscanthus $x$ giganteus on antioxidant capacity of the obtained lignin was studied. Four organosolv lignins extracted with different severity conditions were chosen and tested. Results obtained using the methyl linoleate method have shown a correlation between oxygen uptake index and the combined severity. It was found that lignin extracted at higher severity pre-treatment and with a higher phenolic hydroxyl content, lower aliphatic hydroxyl content, molecular weight and polydispersity has the highest antioxidant capacity.
\end{abstract}

Keywords: Miscanthus x giganteus; Organosolv Lignin; Antioxidant; Combined Severity; Molecular Weight; Phenolic and Aliphatic Hydroxyl

\section{Introduction}

The organosolv pulping has been the subject of considerable research activity and has generated increasing interest as the pulp and paper industry is moving toward minimization of environmental impact [1]. In the 1990's, this technology was developed at the industrial scale in Canada (Alcell ${ }^{\circledR}$ Process). One of the advantages of the organosolv process is the fractionation of the lignocellulosic materials into three major components: cellulosic fibers, hemicelluloses and lignin. As a result, with the increasing attention devoted to the biorefinery concept, a renewed interest in the organosolv treatment is currently observed and this technology seems to be promising for the production of ethanol and high value chemicals and materials from lignin, hemicelluloses and extractives [2].

Indeed, production of high-quality lignin is one of the unique advantages of the organosolv treatment over alternative processes which generally produce a degraded lignin employed in low added value applications and energy production. Oganosolv lignins are high-purity, low molecular weight and sulfur-free products. Moreover, they are soluble in many organic solvents, possess low glass transition temperatures, and are easier to thermally process than kraft lignins. Thus, availability of such highquality lignin in large quantities should stimulate deve-

${ }^{*}$ Corresponding author. lopment in new lignin applications in the fields of fibers, biodegradable polymers, adhesives... Lignins as well as other polyphenols are potent free radical scavengers [3] and organosolv lignins are considered to be a valuable source of antioxidant phenolic compounds, which could be recovered as functional food or feed ingredients. A large number of biochemical or chemical methods are used to measure antioxidant capacity. One class of those methods is based upon inhibition of oxidation of organic substrates: styrene [4], linoleic acid, methyl or ethyl linoleate [5], canola oil [6]. The oxygen uptake measurement is the most direct method used to measure the reaction extent. Moreover, the induced oxidation of methyl linoleate and its radical long chain reaction is well documented and well known to be inhibited by various antioxidants $[7,8]$.

Pan et al. [9] have examined the radical scavenging effect of organosolv lignins from hybrid poplar and the relationship between the antioxidant capacity, lignin structure and extraction conditions. The applicability of lignins from different sources as antioxidants has been also successfully tested [10]. Recently Garcia et al. [11] have studied the effect of pre-treatment processes on the antioxidant capacity of miscanthus sinensis lignins.

Miscanthus $x$ giganteus $(\mathrm{MxG})$ is one of the biomass resources which has attracted considerable attention as a possible dedicated energy crop $[12,13]$. Indeed, $\mathrm{MxG}$ 
presents some valuable advantages: it is a perennial grass which requires little nitrogen fertilizer or herbicide, it can grow to over $3 \mathrm{~m}$ tall per year to produce from 20 to 25 tons of dry matter per hectare, and it is non invasive. Moreover, it is a rhizomatous C4 grass species, which has a high carbon dioxide fixation rate. These properties make miscanthus an interesting raw material for industrial bioconversion processes.

In the present work ethanol organosolv lignins were extracted from Miscanthus x giganteus over a chosen range of severity. The influence of the treatment severity on the antioxidant capacity was studied using the oxygen uptake index method in presence of methyl linoleate.

\section{Experimental}

\subsection{Materials}

The raw Miscanthus $x$ giganteus $(\mathrm{MxG})$ was harvested in spring 2008 in Trier (Germany). The air-dried MxG was milled to a particle size of 1 - $3 \mathrm{~mm}$ using a Wiley mill and stored at room temperature during the course of this study. The untreated feedstock contained 25\% Klason lignin, 37\% cellulose and 36\% hemicelluloses [12]. All chemical reagents used in this study were purchased from Sigma Aldrich and VWR (France) and used as received.

\subsection{Ethanol Organosolv Lignin (EOL) Extraction}

25 g (dry weight, dry matter content about 90\%) of Miscanthus was treated with aqueous ethanol $\left(\mathrm{EtOH} / \mathrm{H}_{2} \mathrm{O}\right.$ $=0.5-0.65)$ in presence of sulfuric acid as a catalyst. The solid to liquid ratio used was 1:8. The pre-treatments were carried out in a $1.0 \mathrm{~L}$ glasslined pressure Parr reactor equipped with a 4842 temperature controller (Parr Instrument Company, Moline, IL). The pre-treated Miscanthus was washed with warm $\left(60^{\circ} \mathrm{C}\right)$ ethanol/water $(8: 1$, $3 \times 50.00 \mathrm{~mL})$. The washes were combined and 3 volumes of water were added to precipitate the Ethanol Organosolv Lignin, which was collected by centrifugation and air dried. EOL characterizations were previously described $[14,15]$.

\subsection{Evaluation of Lignin Antioxidant Properties}

Antioxidant properties of $\mathrm{MxG}$ organosolv lignins were investigated by evaluating oxygen uptake inhibition during oxidation of methyl linoleate. The induced oxidation by molecular oxygen was performed in a gas-tight borosilicate glass apparatus [5,16]. Butan-1-ol was used as solvent for lignin dissolution. Temperature was set to $60^{\circ} \mathrm{C}$, initial conditions inside the vessel were as follows; methyl linoleate (Fluka, 99\%) concentration: $0.32 \mathrm{~mol} / \mathrm{L}$; 2,2'-azobisisobutyronitrile(AIBN) (Fluka, 98\%) concentration: $7.2 \times 10^{-3} \mathrm{~mol} \cdot \mathrm{L}^{-1}$; lignin concentration: 0.2 $\mathrm{g} \cdot \mathrm{L}^{-1}$; oxygen pressure: 145 Torr. Oxygen uptake was monitored continuously by a pressure transducer (Viatron model 104). Without any additive, oxygen uptake is roughly linear and constitutes the control. In the presence of an antioxidant, oxygen consumption is slower, and we estimated the antioxidative capacity of extract by comparing oxygen uptake at a chosen time $(4 \mathrm{~h})$, in the presence of this compound (pressure variation $\Delta$ Psample) and in the absence of the compound ( $\Delta$ Pcontrol) according to:

$$
\text { OUI }=(\Delta \text { Pcontrol }-\Delta \text { Psample }) / \Delta \text { Pcontrol }
$$

This ratio defines antioxidative capacity as an oxygen uptake inhibition index (OUI); it should spread from 0 to $100 \%$, for poor and strong antioxidants, respectively, and may be negative for proxidants. Do not add any kind of pagination anywhere in the paper.

\subsection{Error Analysis}

For all EOL samples, oxygen update index values were calculated from 2 independent experiments performed under the same conditions. Error values (standard deviation) were also estimated following the method described by Mounanga et al. 2008 [16] and are about 5\% for all the essays.

\section{Results and Discussion}

In recent work we have successfully developed an ethanol organosolv process for the pretreatment of $\mathrm{MxG}$ [12] and we have confirmed that this process leads us to produce little degraded and relatively pure ethanol organosolv lignin (EOL) from miscanthus [14]. The isolation of EOL fractions from $\mathrm{MxG}$ is illustrated in Figure 1. Lignin was extracted using various experimental conditions (Table 1), a temperature range between $170^{\circ} \mathrm{C}$ and $190^{\circ} \mathrm{C}$, sulfuric acid (SA) concentration between $0.5 \%$

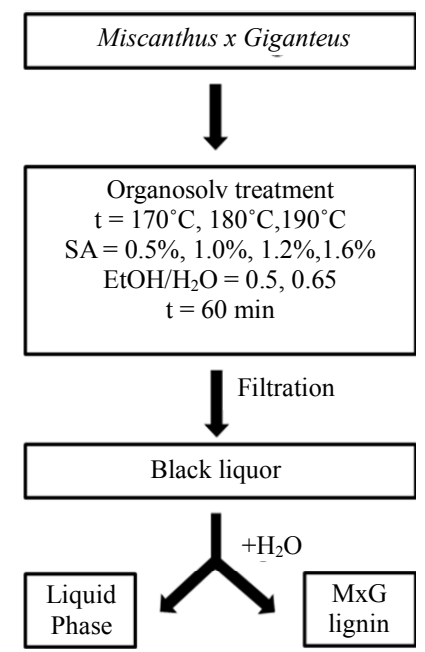

Figure 1. Schematic of the organosolv process. 
Table 1. Lignin oxygen uptake index, phenolic content, molecular weight and polydispersity values.

\begin{tabular}{|c|c|c|c|c|c|c|c|c|c|c|}
\hline Experiment & $\mathrm{T}\left({ }^{\circ} \mathrm{C}\right)$ & $t(\min )$ & $\mathrm{EtOH} / \mathrm{H}_{2} \mathrm{O}(\mathrm{v} / \mathrm{v})$ & $\mathrm{SA}^{1}(\%)$ & $\mathrm{CS}^{2}$ & $\operatorname{OUI}^{3}(\%)$ & $\begin{array}{l}\text { Phenolic OH } \\
\left(\mathrm{mmol} \cdot \mathrm{g}^{-1}\right)\end{array}$ & $\begin{array}{c}\text { Aliphatic } \mathrm{OH} \\
\left(\mathrm{mmol} \cdot \mathrm{g}^{-1}\right)\end{array}$ & $\mathrm{M}_{\mathrm{w}}\left(\times 10^{3}\right)$ & Ip \\
\hline EOL1 & 170 & 60 & 0.65 & 0.5 & 1.75 & 58 & 2.34 & 3.11 & 6.5 & 2.6 \\
\hline EOL2 & 170 & 60 & 0.65 & 1.0 & 2.08 & 62 & 2.72 & 1.78 & 6 & 1.6 \\
\hline EOL3 & 190 & 60 & 0.65 & 1.2 & 2.86 & 86 & 4.04 & 1.07 & 3.6 & 1.4 \\
\hline EOL4 & 190 & 60 & 0.50 & 1.6 & 2.93 & 91 & 3.93 & 1.26 & 3.2 & 1.4 \\
\hline
\end{tabular}

${ }^{1} \mathrm{SA}=$ Sulfuric Acid ${ }^{2} \mathrm{CS}=$ Combined Severity; ${ }^{3} \mathrm{OUI}=$ Oxygen Uptake Index.

and $1.6 \%$, and ethanol concentration of $50 \%$ or $65 \%$, and a reaction time of one hour. The investigation covered a range of combined severity $[(\mathrm{CS})=\log ((\mathrm{t} \exp (\mathrm{T}-\mathrm{Tref}))$ $\mathrm{pH}]$ of $1.75-2.93$; CS describes the severity of the pretreatment as a function of treatment time $(\mathrm{t}=\mathrm{min})$, temperature $\left(\mathrm{T}^{\circ} \mathrm{C}\right)$ and the $\mathrm{pH}$ of the medium[17]. The effect of severity conditions on EOL chemical structure was investigated and published recently [15]. The effect of the treatment severity on miscanthus lignin structure has been also studied and it was demonstrated that the increasing in severity of the organosolv treatment was accompanied by (1) a decrease in the aliphatic $\mathrm{OH}$ groups, (2) an increase of phenolic $\mathrm{OH}$ groups, (3) a decrease of the weight average $(\mathrm{Mw})$ molecular weight and polydispersity and (4) a strong increase in the degree of condensation and a cleavage of $\alpha$ or $\beta$ aryl ether bonds [15].

The study of the antioxidant capacity of EOLs was realized using the induced oxidation method of methyl linoleate. Figure 2 shows the autoxidation of methyl linoleate induced by AIBN-Azobisisobutyronitrile in absence (control) and in presence of lignin (EOL1, EOL2, EOL3, EOL4) extracted at different pre-treatment severities. Antioxidant essays were realized under the same conditions in presence of same lignin concentration $(0.2$ $\left.\mathrm{g} \cdot \mathrm{L}^{-1}\right)$. It appears that the autoxidation of methyl linoleate alone is almost linear [Figure 2 (control)]. By comparing the obtained values in methyl linoleate oxidation, it appears that lignin samples exhibited antioxidant activity by slowing down oxidation of linoleate. This behavior indicates that organosolv lignin acts as a potential anti-oxidant that inhibits the oxidation of methyl linoleate.

Antioxidative capacities [OUI (\%)] defined as the ratio of oxygen uptakes at $4 \mathrm{~h}$ in presence of lignin are also reported in Table 1. As can be seen in this table, results of OUI are not the same for each lignin. These values lead us to classify the antioxidant properties of EOLs from the lowest (OUI $=58 \%)$ to the highest (OUI $=91 \%$ ) as follow EOL1 < EOL2 < EOL3 < EOL4. Furthermore the oxidation of methyl linoleate in presence of a flavonoid polyphenolic antioxidant (catechin) was performed in the same conditions. As a result, miscanthus organosolv lignins recovered at high severity level $(\mathrm{EOL} 3, \mathrm{OUI}=$

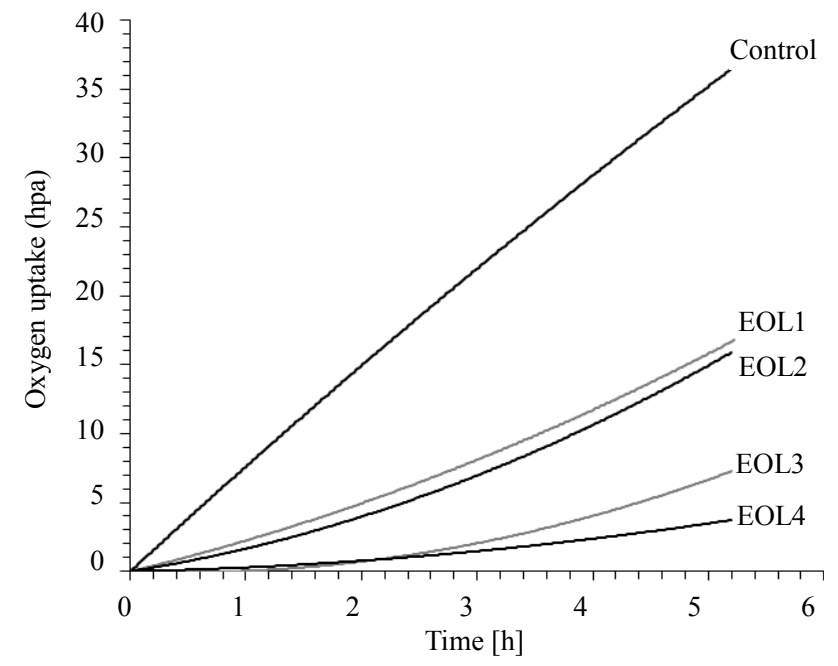

Figure 2. Oxygen uptake during the autoxidation of methyl linoleate induced by AIBN in the presence of MxG organosolv lignins.

$86 \%$ and EOL 4 , OUI $=91 \%$ ) seem to be more efficient than catechin (OUI $=76 \%$ ). As catechin is a very efficient antioxidant in this system [18], more efficient than most of wood extracts, EOLs are very efficient antioxidants.

Several comparisons were performed in order to understand differences of lignin antioxidative capacities and to examine links with lignin structures and experimental condition of processes. Data of combined severity (CS), total phenolic hydroxyl content, total aliphatic hydroxyl content determined by ${ }^{31} \mathrm{P}$ NMR, average molecular weight (Mw) and polydispersity (Ip) determined by GPC [15] are also compiled in Table 1.

Figure 3 shows the Oxygen uptake index (\%) as a function of the combined severity. It can be observed that OUI is positively correlated with the combined severity; the correlation coefficient $\left(\mathrm{R}^{2}\right)$ is about 0.98 . The antioxidant activity increases with the combined severity of the organosolv pretreatment process. Thus the effect of lignin extraction process on its antioxidant properties is confirmed [9-19]. Lignins extracted at elevated temperature, longer reaction time, increased catalyst amount, and dilute ethanol showed higher antioxidant activity. 


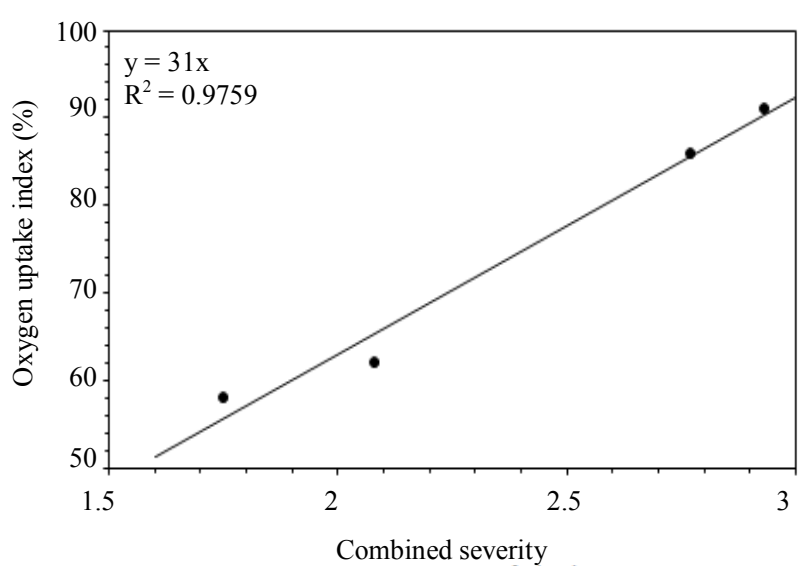

Figure 3. Oxygen uptake index as a function of organosolv pre-treatment combined severity.

The antioxidant efficiency of isolated lignin fractions is described to be related to their structure, purity and polydispersity $[3,9,20]$. Figures 4 (a) and (b) show the oxygen uptake index as a function of phenolic hydroxyl and aliphatic hydroxyl content respectively. As we previously demonstrated, aliphatic hydroxyl moieties in $\mathrm{MxG}$ organosolv lignin decreased with the severity of the treatment while phenolic hydroxyl moieties increased [15]. In our case it can be observed in Figures 4(a) and (b) that OUI is correlated to phenolic hydroxyl group $(\mathrm{ArOH})$ and to aliphatic hydroxyl group (AlkOH) respectively. OUI increases with the lignin phenolic $\mathrm{OH}$ content and with the reduction of aliphatic $\mathrm{OH}$. It is clear from Figures 4(c) and (d) that lignin with high molecular weight and polydispersity had low antioxidant properties. Low molecular weights result from cleavage of some inter-unit bonds in lignin and this degradation is accompanied by an increasing of $\mathrm{OH}$ aliphatic content and decreasing in $\mathrm{OH}$ phenolic group.

The radical scavenging activity of lignin phenolic compounds depends not only on the hydrogen atom withdrawing but also on stability of the radical formed [9]. Figure 5 shows the mechanism generally invoked for induced oxidation of polyunsaturated acids [7-21]. It was also reported that methoxyl groups ameliorate the antioxidant activity [3-9]. In our recent published work results [15], we have shown using ${ }^{13} \mathrm{C}$ NMR that EOLs contain methoxyl group (about $1.4 \mathrm{MeO}$ per aromatic unit) and that no demethoxylation was observed during the organosolv treatment, even at high severity. So the increasing in the antioxidant activity observed in this study could be rationalized by a simultaneous effect: 1) increasing donating hydrogen atom and phenoxyl radical formation due to higher phenolic group contents and 2) stability of the radicals formed in presence of methoxy group in ortho positions (Figure 5).

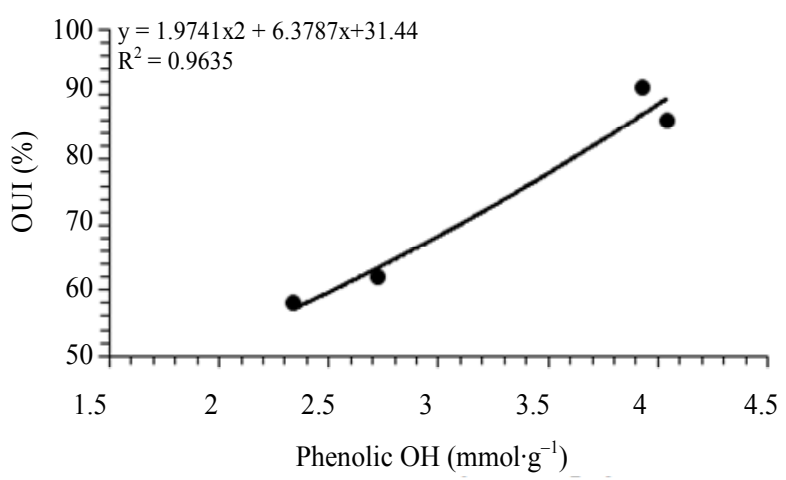

(a)

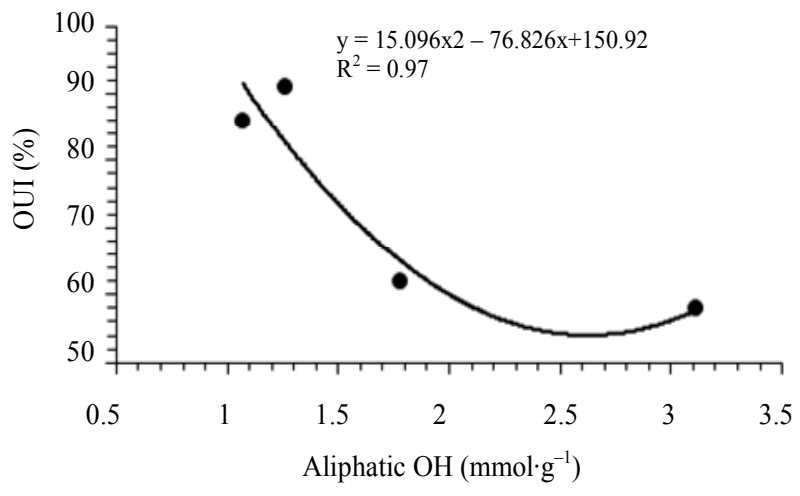

(b)

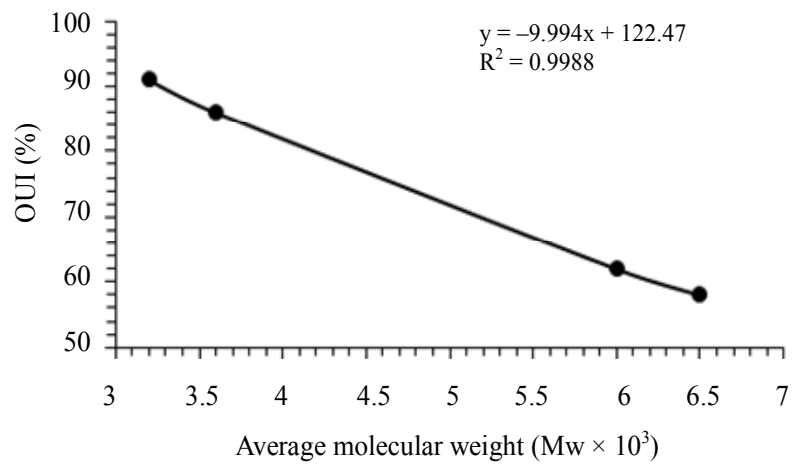

(c)

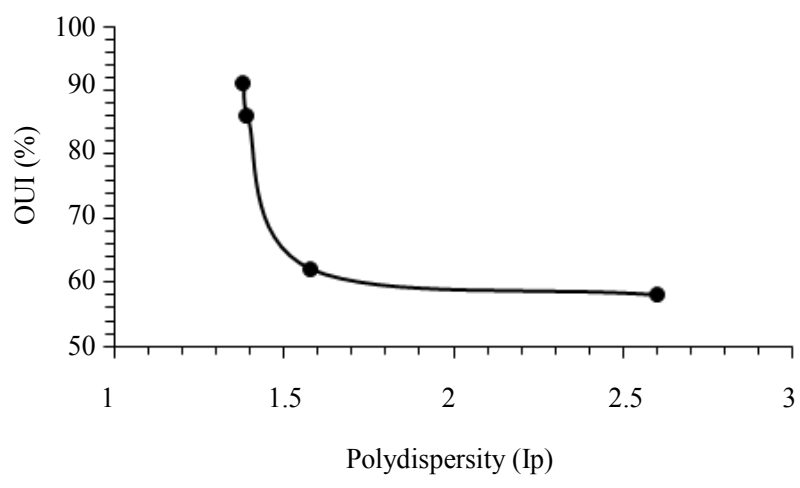

(d)

Figure 4. Oxygen uptake index as a function of EOL phenolic $\mathrm{OH}$ and aliphatic $\mathrm{OH}$ content, average molecular weight and polydispersity. 


$$
\text { AIBN }+\mathrm{LH}+\mathrm{O}_{2} \longrightarrow \mathrm{LO}_{2}+\text { Products }
$$

Figure 5. Mechanism for induced oxidation of polyunsaturated acids.

\section{Conclusion}

In this work it has been shown that Miscanthus $x$ giganteus organosolv lignin have a potential application as antioxidant. Lignin extraction process parameters are important factors that can influence the antioxidant properties. In this way, the obtained results of oxygen updated index have confirmed that lignin antioxidant activity increased positively with the severity treatment. Lignin fractions extracted at elevated temperature and high catalyst contents have revealed a better antioxidant behavior than catechin a well known antioxidant. The present study has also confirmed the close correlation between lignin chemical structure and its antioxidant activities; lignin fractions with higher phenolic hydroxyl groups content, lower molecular weight, polydispersity and aliphatic hydroxyl groups content gave higher values of antioxidant activity.

\section{Acknowledgements}

The authors gratefully acknowledge the financial support of the CPER 2007-2013 "Structuration du Pôle de Compétitivité Fibres Grand'Est” (Competitiveness Fibre Cluster), through regional (Région Lorraine), national (DRRT and FNADT) and European (FEDER) funds.

\section{REFERENCES}

[1] X. B. Zhao, K. K. Cheng and D. H. Liu, "Organosolv Pretreatment of Lignocellulosic Biomass for Enzymatic Hydrolysis," Applied Microbiology and Biotechnology, Vol. 82, No. 5, 2009, pp. 815-827. doi:10.1007/s00253-009-1883-1

[2] A. Ragauskas, C. K. Williams, B. H. Davison, G. Britovsek, J. Cairney, C. A. Eckert, W. J. Frederick Jr., J. P. Hallett, D. J. Leak, C. L. Liotta, J. R. Mielenz, R. Murphy, R. Templer and T. Tschaplinski, "The Path Forward for
Biofuels and Biomaterials," Science, Vol. 311, No. 5760, 2006, pp. 484-489. doi:10.1126/science. 1114736

[3] T. Dizhbite, G. Telysheva, V. Jurkjane and U. Viesturs, "Characterization of the Radical Scavenging Activity of Lignins-Natural Antioxidants," Bioresource Technology, Vol. 95, No. 3, 2004, pp. 309-317.

doi:10.1016/j.biortech.2004.02.024

[4] G. W. Burton, T. Doba, E. Gabe, L. Hughes, F. L. Lee, L. Prasad and K. U. Ingold, "Autoxidation of Biological Molecules. 4. Maximizing the Antioxidant Activity of Phenols," Journal of the American Chemical Society, Vol. 107, No. 24, 1985, pp. 7053-7065.

[5] H. Eloualja, D. Perrin and R. Martin, "Kinetic Study of the Thermal Oxidation of All-Trans- $\beta$-Carotène and Evidence of Its Antioxigen Properties," New Journal of Chemistry, Vol. 19, No. 11, 1995, pp. 863-872.

[6] U. N. Wanasundara and F. Shahidi, "Stabilization of Canola Oil with Flavonoids," Food Chemistry, Vol. 50, No. 4, 1994, pp. 393-396. doi:10.1016/0308-8146(94)90211-9

[7] C. Rousseau, C. Richard and R. Martin, "Influence Inhibitrice Comparé des Vitamines $\mathrm{C}$ et $\mathrm{E}$ sur l'Oxydation Induite du Linoléate de Methyl vers $80^{\circ} \mathrm{C}$ et Effet de Synergie," Chimie Physique, Vol. 80, No. 11-12, 1983, pp. 827-829.

[8] N. Uri, "Physico-Chemical Aspects of Autoxidation," In: W. O. Lundberg, Ed., Autoxidation and Antioxidants, Inter-Science Publishers, New York, 1961.

[9] X. J. Pan, J. F. Kadla, K. Ehara, N. Gilkes and J. N. Saddler, "Organosolv Ethanol Lignin from Hybrid Poplar as a Radical Scavenger: Relationship between Lignin Structure Extraction Conditions and Antioxidant Activity," Journal of Agriculture and Food Chemistry, Vol. 54, No. 16, 2006, pp. 5806-5813. doi:10.1021/jf0605392

[10] V. Urgatondo, M. Mitjans and M. P. Vinardell, “Applicability of Lignins from Different Sources as Antioxidants Based on the Protective Effects on Lipid Peroxidation Induced by Oxygen Radicals," Industrial Crops and Products, Vol. 30, No. 2, 2009, pp. 184-187. doi:10.1016/j.indcrop.2009.03.001

[11] A. Garcia, A. Toledano, M. A. Andres and J. Labidi, "Study of the Antioxidant Capacity of Miscanthus Sinensis Lignins," Process Biochemistry, Vol. 45, No. 6, 2010, pp. 935-940. doi:10.1016/j.procbio.2010.02.015

[12] N. Brosse, P. Sannigrahi and A. Ragauskas, "Pretreatment of Miscanthus $x$ giganteus Using the Ethanol Organosolv Process for Ethanol Production," Industrial \& Engineering Chemistry Research, Vol. 48, No. 18, 2009, pp. 8328-8334. doi:10.1021/ie9006672

[13] A. Sørensen, P. J. Teller, T. Hilstrom and B. K. Ahring, "Hydrolysis of Miscanthus for Bioethanol Production Using Dilute Acid Presoaking Combined with Wet Explosion Pre-Treatment and Enzymatic Treatment," Bioresource Technology, Vol. 99, No. 14, 2008, pp. 6602-6607. doi:10.1016/j.biortech.2007.09.091

[14] R. El. Hage, N. Brosse, L. Chrusciel, C. Sanchez, P. Sannigrahi and A. Ragauskas, "Characterization of Milled Wood Lignin and Ethanol Organosolv Lignin from Miscanthus," Polymer Degradation and Stability, Vol. 94, No. 
10, 2009, pp. 1632-1638.

doi:10.1016/j.polymdegradstab.2009.07.007

[15] R. El Hage, N. Brosse, P. Sannigrahi and A. Ragauskas, "Effect of Process on the Chemical Structure of Miscanthus Ethanol Organosolv Lignin," Polymer Degradation and Stability, Vol. 95, No. 6, 2010, pp. 997-1003. doi:10.1016/j.polymdegradstab.2010.03.012

[16] T. K. Mounanga, P. Gérardin, B. Poaty, D. Perrin and C. Gérardin, "Synthesis and Properties of Antioxidant Amphiphilic Ascorbate Salts," Colloids and Surfaces A: Physicochemical Engineering Aspects, Vol. 318, No. 1-3, 2008, pp. 134-140. doi:10.1016/j.colsurfa.2007.12.048

[17] D. Montane, X. Farriol, J. Salvado, P. Jollez and E. Chornet, "Fractionation of Wheat Straw by Steam-Explosion Pre-Treatment and Alkali Delignification. Cellulose Pulp and Byproducts from Hemicelluloses and Lignin," Journal of Wood Chemistry and Technology, Vol. 18, No. 2, 1998, pp. 171-191. doi:10.1080/02773819809349575
[18] P.-N. Diouf, A. Merlin and D. Perrin, "Antioxidant Properties of Wood Extracts and Colour Stability of Woods," Annals of Forest Science, Vol. 63, No. 5, 2006, pp. 525-534. doi:10.1051/forest:2006035

[19] H. Nadji, P.-N. Diouf, A. Benaboura, Y. Bedard, B. Riedl and T. Stevanovic, "Comparative Study of Lignins Isolated from Alfa Grass (Stipa tenacissima L.)," Biorsource Technology, Vol. 100, No. 14, 2009, pp. 3585-3592. doi:10.1016/j.biortech.2009.01.074

[20] C. Pouteau, P. Dole, B. Cathala, L. Averous and N. Boquillon, "Antioxidant Properties of Lignin in Polypropylene," Polymer Degradation and Stability, Vol. 81, No. 1, 2003, pp. 9-18. doi:10.1016/S0141-3910(03)00057-0

[21] E. Niki, T. Saito, A. Kawakami and Y. Kamiya, "Inhibition of Oxidation of Methyl Linoleate in Solution by Vitamin E and Vitamin C," The Journal of Biological Chemistry, Vol. 259, No. 7, 1984, pp. 4177-4182. 